\title{
Post-surgical pyoderma gangrenosum following electrosurgery and elliptical excision: A rare disease following common procedures
}

\section{Mario Gaston Toledo Lelevier, Raul Gerardo Mendez Flores, Luzma Kareena Jimenez Leon, Teresa Aranzazu Gomez Cevallos, Marisol Ramirez Padilla}

Universidad De Guadalajara, Dermatology Service of Civil Hospital of Guadalajara "Fray Antonio Alcalde", Guadalajara, Mexico

Corresponding author: Raul Gerardo Mendez Flores, MD, E-mail: mendezfloresdr@gmail.com

\begin{abstract}
Pyoderma gangrenosum (PG) is a rare neutrophilic dermatosis with an estimated annual incidence of 3-10 cases per million per year. Post-surgical pyoderma gangrenosum (PSPG) refers to the development of pyoderma gangrenosum at surgical sites in the postoperative period. A 55 -year-old female was admitted with two acute and enlarging painful breast ulcerations resistant to antibiotic treatment following surgical resection and electrofulguration by her dermatologist. Multiple wound cultures were negative. A skin biopsy was consistent with PG and the wounds improved after adequate oral immunosuppressive therapy. A high index of suspicion, exclusion of other differential diagnoses, and previous experience with this disease ensured an early diagnosis, prevented injury to life and unnecessary debridement, and resulted in an excellent outcome for the patient.
\end{abstract}

Key words: Pyoderma gangrenosum; Breast; Elliptical excision; Electrosurgery

\section{INTRODUCTION}

Pyoderma gangrenosum (PG) is a rare neutrophilic dermatosis characterized by a well-defined painful ulcer with a red-violaceus border. The annual incidence is estimated at 3-10 cases per million per year [1]. The peak incidence occurs between the ages of 20-50 years, with a possible slight female preponderance and with approximately $4 \%$ of patients being children [2]. There are immunological alterations involved and various well-recognized associations, such as rheumatological diseases, inflammatory bowel disease, and hematological neoplasms, present in 50-70\% of cases [3]. Post-surgical pyoderma gangrenosum (PSPG) refers to the development of pyoderma gangrenosum at surgical sites in the postoperative period. Compared to PG, PSPG occurs in uncommon locations and is less likely to be associated with systemic disease. Herein, we present a case of PSPG triggered by common dermatologic procedures.

\section{CASE REPORT}

A 55-year-old female presented herself to our clinic with two acute and enlarging painful breast ulcerations. The past medical history was unremarkable. No evidence of rheumatologic disease or malignancy was found. A physical examination revealed two undermined ulcers, $3 \mathrm{~cm} \times 1.5 \mathrm{~cm}$ and $3 \mathrm{~cm} \times 2 \mathrm{~cm}$ in size, respectively, with well-defined purplish erythematous edges and seropurulent discharge, and were located on the right breast in the inframammary area (Fig. 1) and on the lower outer quadrant of the left breast (Fig. 2). Three weeks ago, she had a surgical resection of an atypical melanocytic nevus in the left mammary region and electrofulguration of a seborrheic keratosis in the

\footnotetext{
How to cite this article: Toledo Lelevier MG, Mendez Flores RG, Jimenez Leon LK, Gomez Cevallos TA, Ramirez Padilla M. Post-surgical pyoderma gangrenosum following electrosurgery and elliptical excision: A rare disease following common procedures. Our Dermatol Online. 2021;12(1):54-57.

Submission: 16.11.2020; Acceptance: 05.12.2020

DOI: 10.7241/ourd.20211.14
} 


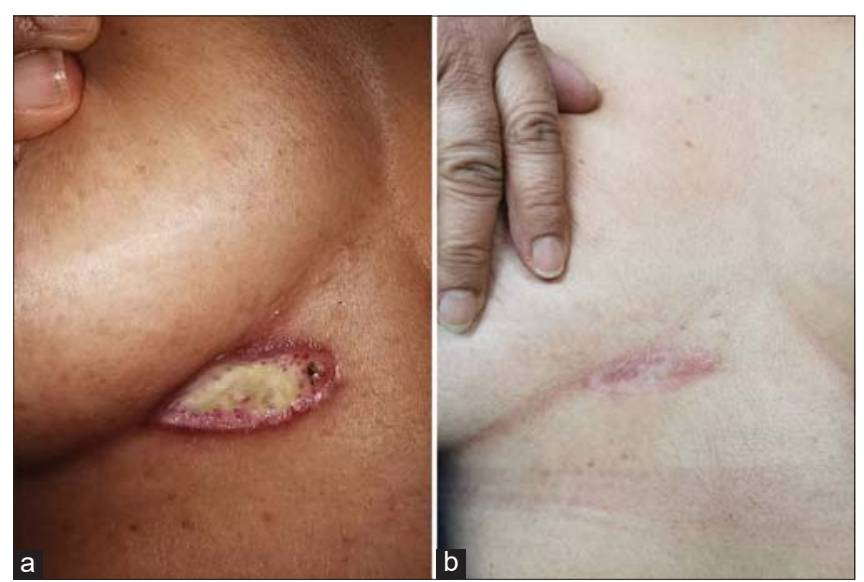

Figure 1: (a) The initial lesion located in the right inframammary area consisting of a purulent ulcer with well-demarcated violaceous edges. (b) The lesion after four months of treatment (ulcer resolution with a cribriform scar).

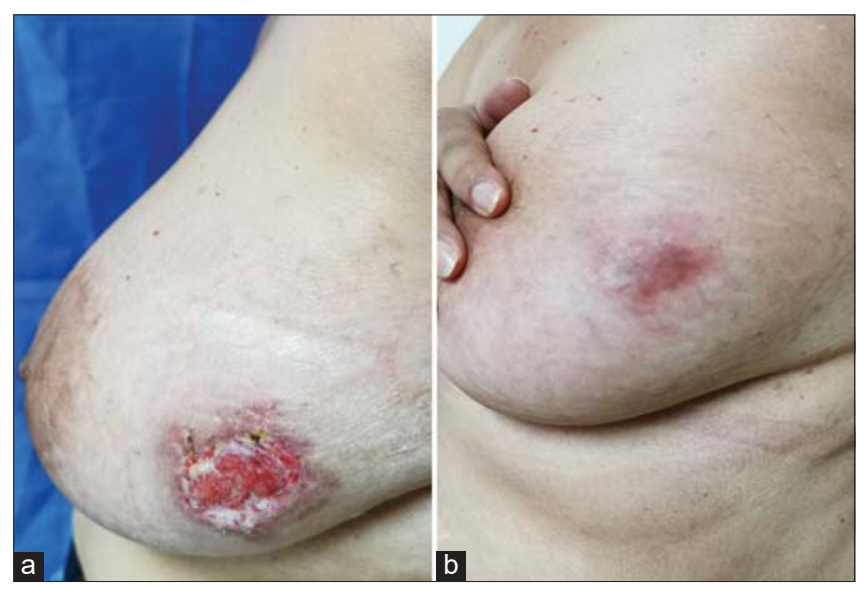

Figure 2: (a) The initial lesion located in the left mammary area consisting of a purulent ulcer with central exudate and violaceous edges on an erythematous background. (b) The lesion after four months of treatment (ulcer resolution with residual scar).

right inframammary area. Postoperative infection was suspected, and the patient was started on topical and systemic antibiotics with no improvement and progressive worsening of the ulcers. Cultures from the ulceration discharge grew no bacteria, acid-fast bacilli, or fungi. A punch biopsy revealed ulceration and a superficial neutrophilic inflammatory infiltrate in the dermis, compatible with the diagnosis of early PG (Fig. 3). Treatment was started with prednisone $60 \mathrm{mg} /$ day and azathioprine $100 \mathrm{mg}$ /day with an excellent response at 2 months and a subsequent tapering regimen with no relapse at 18 months.

\section{DISCUSSION}

PG was first described by French dermatologist Brocq in 1916, although it was not until 1930 that it was given

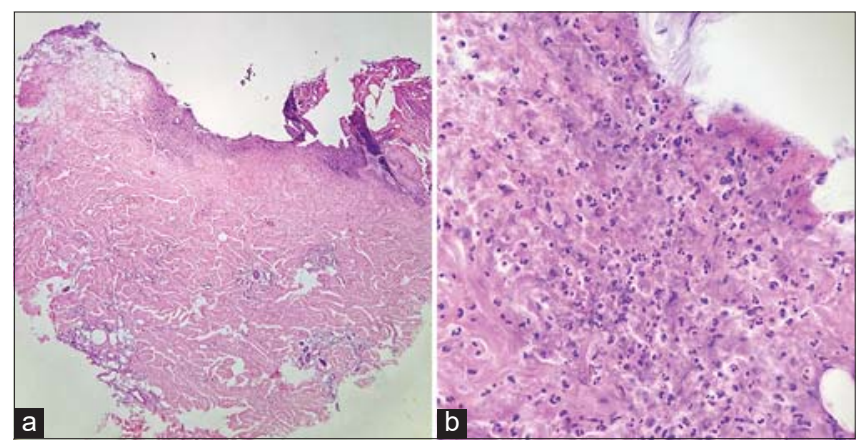

Figure 3: (a) Necrosis with ulceration of the epidermis (H\&E, 40x). (b) High-power examination revealing dense neutrophilic infiltrate in the dermis; no bacterial components or tumor cells were observed (H\&E, 200x).

its present name by Burnsting at the Mayo Clinic under the assumption that it was an infectious disease [4]. The pathogenesis of the disease is unknown, but the proposed causes include immune-complex mediated neutrophilic vascular reactions.

Currently, three factors are considered to be involvedneutrophilic dysfunction, genetic susceptibility, and systemic inflammation-all of which favor an important immunological alteration in the host. This systemic inflammation has been demonstrated with a high presence of IL-8, IL-23, and TNF-alpha. Unquestionably, the most significant triggering factor is pathergy (Koebner phenomenon), reported in 20-30\% of patients affected by PG. Any kind of skin trauma due to surgery, injections, prick tests, or insect bites may induce the appearance of a new lesion or worsen preexisting ones [4].

Around $50 \%$ of patients with PG have an associated underlying disease. This was confirmed by a cohort study on 356 patients, $45 \%$ of which had preexisting conditions; $41 \%$ with inflammatory bowel disease, $21 \%$ with inflammatory arthritis, $11 \%$ with hematological alterations ranging from different types of leukemias, monoclonal gammopathies, and myelodysplastic syndrome, and finally $7 \%$ with solid organ neoplasms [5].

PSPG is also known as postoperative PG or pathergic PG. Clinical presentation usually begins as multiple small areas of dehiscence or ulceration several days to weeks after the procedure. They exhibit erythema and severe pain, followed by wound dehiscence and the formation of an aggressive ulcer. The trauma caused by surgery, and consequent cytokine release, is likely the initiating factor of pathergy in PG [6]. Compared to classic PG, PSPG is associated with systemic disease in only $22-35 \%$ of cases, with the most common 
comorbidity being myeloproliferative disorders [7]. This leaves most patients with no predisposing comorbidity other than surgery.

Although cases of PSPG reported in the literature remain rare, several locations have been reported, following surgical incisions almost anywhere on the body. In a study of 220 patients identified with PSPG, the most common procedures were breast (25\%), cardiothoracic (14\%), abdominal (14\%), and obstetric (13\%) surgeries. The most common breast procedures in which post-surgical pyoderma gangrenosum was reported were bilateral reduction mammoplasty (45\%), breast reconstruction (25\%), and other procedures $(12.5 \%)$, such as excision or biopsy, as in our case [6].

In a clinical scenario of PSPG, the major and minor diagnostic criteria for PG [8] are scarcely useful. The rapid progression of lesions and severe pain are inconstant in PSPG. Only some patients experience severe pain and breast tenderness after breast surgery in the early phase of PSPG. Moreover, there may be no previous history of $\mathrm{PG}$, both as in our case and as in those reported in the literature $[9,10]$.

Due to its similar presentation to wound infection, PSPG is a complex diagnosis that may be challenging to identify in a context of recent surgery. Therefore, as our case illustrates, PG should be considered in the setting of non-healing postoperative wounds refractory to antibiotics. Misdiagnosis of PSPG may lead to ineffective treatment with antibacterial agents and debridement, which may accelerate the progression of the disease. Thus, it is imperative to make a prompt diagnosis to reduce its destructive course.

In the initial phase, histological examination may show perivascular lymphocytic infiltrate and intradermal abscess formation. As the disease progresses, significant edema, intense neutrophilic infiltrate, necrosis, and granuloma formation are found in the dermis, as well as vasculitis with or without leukocytoclasia. However, none of these findings are pathognomonic [11]. Differential diagnoses are diverse since many diseases produce ulcers, mainly of infectious, neoplastic, and vascular etiologies.

A review of the literature demonstrated no consensus for the treatment of PSPG. The most commonly employed protocol involves high-dose systemic corticosteroids, such as oral prednisone, prednisolone, and intravenous methylprednisolone. Since the complete resolution of an ulcer takes months, in order to maintain the response once the glucocorticoid is stopped, it is recommended to associate a steroid-sparing agent with the treatment; these include mycophenolate mofetil 2-3 g/day, azathioprine 100-300 mg/day, or methotrexate 10-25 mg/week [12]. In our case, we observed an excellent response with a combination of prednisone and azathioprine.

Systemic cyclosporine is an alternative first-line treatment for patients who cannot tolerate systemic glucocorticoid therapy [13]. The second-line treatment is biological therapy with infliximab, etanercept, and adalimumab, the latter with a better clinical response, mainly in patients with rheumatoid arthritis [12]. Other therapies described are dapsone 50-200 mg/day or minocycline $200 \mathrm{mg}$ /day in a context of limited disease without corticosteroids or adjuvant to them. With refractory disease, starting treatment with intravenous immunoglobulin or cyclophosphamide should be considered [14].

The course of the disease is chronic, with frequent relapses and exacerbations, for which close follow-up must be observed and paraclinical monitoring and systemic therapy should be adjusted if necessary.

\section{CONCLUSION}

PSPG must be considered in any patient undergoing surgery who afterward manifests characteristic necrotic lesions with delayed wound healing, fever, and severe localized pain.

Considering pyoderma gangrenosum as an underdiagnosed disease, clinical suspicion aids a timely diagnosis and completes the diagnostic approach that may unmask this pathology. In our case, timely diagnosis avoided detrimental procedures for the patient, such as surgical debridement, and prevented further disease progression.

\section{Consent}

The examination of the patient was conducted according to the principles of the Declaration of Helsinki.

The authors certify that they have obtained all appropriate patient consent forms, in which the patients gave their consent for images and other clinical information to be included in the journal. The patients understand that their names and initials will not be published and due effort will be made to conceal their identity, but that anonymity cannot be guaranteed. 


\section{REFERENCES}

1. Ruocco E, Sangiuliano S, Gravina AG, Miranda A, Nicoletti G. Pyoderma gangrenosum: An updated review. J Eur Acad Dermatol Venereol. 2009;23:1008-17.

2. Bhat RM. Pyoderma gangrenosum: An update. Indian Dermatol Online J. 2012;3:7-13.

3. Powell F, Hackett B, Wallach D. Pyoderma gangrenosum. In: Goldsmith L, Katz S, Gilchrest B, ed. Fitzpatrick's Dermatology In General Medicine. 8th ed. New York: McGraw Hill; 2012:371.

4. Cozzani E, Gasparini G, Parodi A. Pyoderma gangrenosum: A systematic review. G Ital Dermatol Venereol. 2014;149:587-600.

5. Ashchyan HJ, Butler DC, Nelson CA, Noe MH, Tsiaras WG, Lockwood SJ, et al. The Association of Age With Clinical Presentation and Comorbidities of Pyoderma Gangrenosum [published correction appears in JAMA Dermatol. 2018;154:630]. JAMA Dermatol. 2018;154:409-13.

6. Zuo KJ, Fung E, Tredget EE, Lin AN. A systematic review of post-surgical pyoderma gangrenosum: Identification of risk factors and proposed management strategy. J Plast Reconstr Aesthet Surg. 2015;68:295-303.

7. Tolkachjov SN, Fahy AS, Cerci FB, Wetter DA, Cha SS, Camilleri MJ. Postoperative pyoderma gangrenosum: A clinical review of published cases. Mayo Clin Proc. 2016;91:1267-79.

8. Su WP, Davis MD, Weenig RH, Powell FC, Perry HO. Pyoderma gangrenosum: Clinicopathologic correlation and proposed diagnostic criteria. Int J Dermatol. 2004;43:790-800.

9. Ouazzani A, Berthe JV, de Fontaine S. Post-surgical pyoderma gangrenosum: a clinical entity. Acta Chir Belg. 2007;107:424-8.

10. Horner B, El-Muttardi N, Mercer D. Pyoderma gangrenosum complicating bilateral breast reduction. BrJ Plast Surg. 2004;57:679-81.

11. Weedon D, Hurt M. The vasculopathic reaction pattern. In: Weedon'S Skin Pathology. 3rd ed. London: Elsevier; 2010:195.

12. Patel F, Fitzmaurice S, Duong C, He Y, Fergus J, Raychaudhuri SP, et al. Effective strategies for the management of pyoderma gangrenosum: A comprehensive review. Acta Derm Venereol. 2015;95:525-31.

13. Ahronowitz I, Harp J, Shinkai K. Etiology and management of pyoderma gangrenosum: A comprehensive review. Am J Clin Dermatol. 2012;13:191-211.

14. Din RS, Tsiaras WG, Li DG, Mostaghimi A. Efficacy of systemic dapsone treatment for pyoderma gangrenosum: A retrospective review. J Drugs Dermatol. 2018;17:1058-60.

Copyright by Mario Gaston Toledo Lelevier, et al. This is an open-access article distributed under the terms of the Creative Commons Attribution License, which permits unrestricted use, distribution, and reproduction in any medium, provided the original author and source are credited.

Source of Support: Nil, Conflict of Interest: None declared. 